\title{
Comportamentos associados à manipulação de domissanitários
}

\author{
Behavior associated with the handling of sanitizers \\ Comportamientos asociados a la manipulación de productos de limpeza domésticos
}

Recebido: 17/03/2021 | Revisado: 23/03/2021 | Aceito: 28/03/2021 | Publicado: 09/04/2021

Talita Ferreira do Nascimento ORCID: https://orcid.org/0000-0002-3079-0950 Centro Universitário Nossa Senhora do Patrocínio, Brasil E-mail: talita.alferreira@gmail.com

José Teixeira de Seixas Filho ORCID: https://orcid.org/0000-0002-5021-1290

Centro Universitário Augusto Motta, Brasil E-mail: seixasfilho@yahoo.com.br

Gustavo Henrique Oliveira da Rocha ORCID: https://orcid.org/0000-0001-9158-1194 Universidade de São Paulo, Brasil E-mail: gustavohrocha@gmail.com

Marcus Tolentino Silva ORCID: https://orcid.org/0000-0002-7186-9075 Universidade de Sorocaba, Brasil E-mail: marcus.silva@prof.uniso.br

Rômulo Tadeu Dias de Oliveira ORCID: https://orcid.org/0000-0002-3352-106X Universidade de Sorocaba, Brasil E-mail: romulo.oliveira@prof.uniso.br Éric Diego Barioni

ORCID: https://orcid.org/0000-0003-3477-7072 Universidade de Sorocaba, Brasil E-mail: eric.barioni@prof.uniso.br

\begin{abstract}
Resumo
Este estudo buscou avaliar os comportamentos associados à manipulação de domissanitários em um bairro predominantemente de baixa renda, localizado na cidade de Itu - SP, Brasil. Trata-se de um inquérito baseado em um questionário semiestruturado, individual e fechado aplicado a 100 moradores do bairro Parque São Camilo com idade igual ou superior a 30 anos. A extensão das associações entre os fatores avaliados foi determinada por teste exato de Fisher, e associações foram consideradas significativas quando $\mathrm{p}<0,05$. Desinfetantes e amaciantes de tecidos foram os domissanitários mais utilizados pela população. Independentemente da idade, sexo, histórico de intoxicações e uso de EPI, o comportamento de ler o rótulo das embalagens mostrou-se associado à lavagem das mãos e ao não uso de produtos saneantes clandestinos. Pode-se inferir que políticas públicas de conscientização da população local ou o letramento da população possam ser os responsáveis pelos hábitos seguros de manejo de domissanitários e não uso de produtos clandestinos.
\end{abstract}

Palavras-chave: Saneantes; Intoxicações domésticas; Rotulagens.

\begin{abstract}
This study aimed to evaluate behaviors associated with handling of sanitizers in a largely low-income neighborhood located in Itu - SP, Brazil. This work was carried out based upon application of a semi-structured, individual and closed questionnaire to 100 inhabitants of Parque São Camilo aged 30 or older. The extent of the associations between the assessed factors was determined by Fisher exact and these were considered significant when $p<0.05$. Disinfecting products and fabric softeners were the sanitizing agents most often used by the population. Regardless of age, sex, previous history of intoxications and use of personal protective equipment, the habit of reading product labels was associated with handwashing and not using clandestine products. It can be inferred that either public policies aimed at conscientizing the local population or their literacy might be the reason for the assessed population having safe sanitizer handling habits and not using clandestine products.
\end{abstract}

Keywords: Sanitizing agents; Domestic intoxications; Labeling.

\section{Resumen}

Este estudio buscó evaluar los comportamientos asociados con el manejo de productos de limpieza del hogar en un barrio predominantemente de baja renta, ubicado en la ciudad de Itu - SP, Brasil. Se trata de una encuesta basada en un cuestionario semiestructurado, individual y cerrado aplicado a 100 habitantes del barrio Parque São Camilo con 30 
años o más. La extensión de las asociaciones entre los factores evaluados se determinó mediante a la prueba exacta de Fisher, y las asociaciones se consideraron significativas cuando $\mathrm{p}<0,05$. Los desinfectantes y suavizantes para ropa fueron los productos de limpieza del hogar más utilizados por la población. Independientemente de la edad, el sexo, los antecedentes de intoxicación y el uso de EPI, se demostró que el comportamiento de leer la etiqueta en el empaque estaba asociado con el lavado de manos y la no utilización de productos desinfectantes clandestinos. Se puede inferir que las políticas públicas de sensibilización de la población local o la alfabetización de la población pueden ser responsables de los hábitos de manejo seguro de los agentes de limpieza del hogar y no del uso de productos clandestinos.

Palabras clave: Higienizante; Intoxicaciones domésticas; Etiquetado.

\section{Introdução}

Domissanitários, como amaciantes, detergentes, ceras líquidas, polidores, limpa-vidros, limpa-pneus, desinfetantes, sabonetes, entre outros, são produtos saneantes fabricados pela indústria, e sua utilização visa a higienização, desinfecção ou desinfestação de áreas domiciliares ou públicas. Estes produtos químicos, ainda que sejam tóxicos, fazem parte do dia a dia dos brasileiros, e podem conter substâncias perigosas, como soda cáustica, potássica, fenóis, cresóis e hidrocarbonetos substâncias que são extremamente nocivas à saúde - e que podem causar casos graves de intoxicação (Souza et al., 2015; Barioni \& Profeta, 2021).

Não obstante, produtos saneantes de fabricação caseira, produzidos sem qualquer controle de qualidade, são vendidos livremente em feiras e no comércio popular. Tais produtos não possuem rótulos e informações básicas sobre boas práticas de fabricação e segurança ao consumidor, e por este motivo estes produtos clandestinos oferecem riscos adicionais à saúde da população e ao meio ambiente (ANVISA, 2013; Pinheiro et al., 2014).

Os rótulos de produtos saneantes domissanitários compreendem uma fonte segura de informações ao consumidor, e sua leitura antes do uso do produto garante a segurança do manipulador e das pessoas e animais que estão próximos do ambiente que foi exposto ao produto. A Agência Nacional de Vigilância Sanitária (ANVISA) exige que, no mínimo, os rótulos de produtos domissanitários possuam as seguintes informações: nome do produto, componente ativo ou princípio ativo, lote, validade, advertências, dados completos do fabricante, incluindo razão social, CNPJ, químico responsável, número no Conselho Regional de Química (CRQ) e registro na ANVISA, e o número de telefone do Serviço de Atendimento ao Consumidor (SAC). Deve-se encontrar nos rótulos também as frases: "Produto Notificado na Anvisa" ou o número do registro no Ministério da Saúde (MS); "Antes de usar leia as instruções do rótulo", para que o consumidor saiba como utilizar o produto e, ainda, avisos sobre os perigos, informações de primeiros socorros e o número de telefone do disque intoxicação da ANVISA (ANVISA, 2005; 2010; 2012; 2020).

Crianças na faixa etária de 01 a 04 anos são particularmente mais vulneráveis aos acidentes domésticos envolvendo a intoxicação por saneantes domissanitários, e uma série de fatores, como embalagens atrativas contendo conteúdo colorido, aspecto muitas vezes leitoso e a curiosidade das crianças, associados ao uso irresponsável, em partes, por um armazenamento inadequado, incrementam o número de casos de intoxicação por domissanitários na infância (Presgrave, Camacho \& Villas Boas, 2009; Rodrigues, 2010; Costa et al., 2020; Rodrigues et al., 2021; Barioni \& Profeta, 2021).

Por outro lado, a não leitura de rótulos e o não uso ou o uso inadequado ou insuficiente de EPI garantem que as crianças não sejam as únicas vítimas em potencial. A segunda e terceira faixa etária mais acometida pelos acidentes com saneantes domissanitários compreendem indivíduos com idade entre 20 a 39 anos e 40 a 59 anos, respectivamente (Brasil, 2020; Barioni \& Profeta, 2021; Almeida et al., 2021). Neste sentido, ao buscar compreender comportamentos de riscos, como a não leitura de rótulos, descarte e armazenamento inadequado de produtos e embalagens, e utilização de produtos clandestinos, buscamos realizar esta pesquisa em um bairro predominantemente de baixa renda de uma cidade localizada no interior do Estado de São Paulo. 


\section{Metodologia}

Este trabalho entrevistou 100 moradores do bairro Parque São Camilo, que está localizado na cidade de Itu - SP, Brasil. Os participantes assinaram termo de consentimento livre e esclarecido (TCLE) autorizando sua participação na pesquisa e assumindo os riscos mínimos de participação na mesma. O trabalho foi submetido e aprovado pelo Comitê de Ética em Pesquisa (CEP) da Universidade de Sorocaba sob o número: 3.204.959.

Buscando avaliar a segunda e terceira faixa etária mais acometida pelos casos de intoxicação, foram recrutados homens e mulheres com idade igual ou superior a 30 anos. Indivíduos mais jovens, com idade entre 20 e 29 anos, não foram entrevistados, uma vez que fatores como a educação superior, a inserção no mercado de trabalho, bem como o fato de muitos jovens adultos ainda morarem com os pais, podem reduzir a permanência em casa e a realização de afazeres domésticos por estes indivíduos. Este estudo abrangeu uma grande área do bairro, e buscou analisar a utilização de produtos domissanitários, investigando suas múltiplas dimensões.

Foram observadas informações sobre rotulagem, estocagem e aplicação de produtos de limpeza em uma localidade predominantemente de baixa renda. A coleta de dados foi realizada a partir de um instrumento de aproximação realizado por questionário semiestruturado, individual e fechado, que foi adaptado do trabalho de Souza e colaboradores (2015) e aplicado pelos autores, cujas questões identificaram a percepção das situações de vulnerabilidade da população pelo uso inadequado destes produtos, coletando fatos relacionados às práticas que envolvam o manuseio de substâncias químicas habitualmente utilizadas nas residências.

A partir dos dados gerados de tabulação das respostas obtidas, foi realizada uma análise qualitativa descritiva. Avaliou-se o percentual de pessoas que se utilizam de determinados tipos de produtos domissanitários, seus manejos em diferentes aplicabilidades e o seu descarte para o meio ambiente, sendo utilizado como critério de avaliação os seguintes questionamentos: (a) quais são os produtos químicos domésticos utilizados; (b) quais as aplicações dos produtos químicos na manutenção do lar; (c) como o usuário manuseia o produto químico adquirido para a manutenção do lar; (d) qual é o nível de consciência quanto ao impacto causado pelo seu descarte na natureza. Essas categorias possibilitaram a estruturação de um conjunto de conceitos articulados entre si, constituindo assim um instrumento válido para o desenvolvimento do tema da pesquisa.

Em segundo momento, foi realizada verificação de possíveis associações entre as variáveis qualitativas nominais assumindo como variável independente a leitura ou não de rótulos de produtos domissanitários e como variáveis dependentes sexo, idade, uso de EPIs, histórico de intoxicação prévia por domissanitários e hábito conjunto de lavagem de mãos e uso de produtos clandestinos. A extensão das associações foi avaliada por teste exato de Fisher, e associações foram consideradas significativas quando $\mathrm{p}<0,05$. Análises foram realizadas utilizando o software GraphPad Prism $7^{\circledR}$. Os autores GHOR e MTS forneceram suporte metodológico para realização de análises estatísticas.

\section{Resultados}

A análise dos dados foi realizada a partir das respostas que foram obtidas por meio da aplicação de questionários. Dos 100 moradores entrevistados, 39 eram homens e 61 eram mulheres. Dos sujeitos de pesquisa, independentemente do sexo, 40 tinham idade entre 30 e 39 anos; 29 tinham idade entre 40 e 49 anos; 21 tinham idade entre 50 e 59 anos e, 10 tinham idade igual ou superior a 60 anos. Com os números que foram demonstrados acima, é possível verificar a especificidade de nossa amostra que compreendeu faixas etárias excetuando-se as de 20 a 29 anos, e a infantil, de 01 a 04 anos. Ainda que a faixa etária infantil seja a mais afetada pelos quadros de intoxicação envolvendo saneantes domissanitários, as faixas etárias englobadas em nosso estudo são também bastante passíveis de sofrerem intoxicações e são justamente hábitos de manuseio desta população que predispõe que crianças possam também sofrer intoxicações. 
Nossos resultados mostraram que os produtos domissanitários mais utilizados nos lares do bairro Parque São Camilo em Itu foram: os desinfetantes (100\%), amaciante para tecidos (95\%), alvejante (83\%), mata-mosquitos em aerossol (68\%), sapólio (30\%), produto contra ratos (15\%) e soda cáustica (14\%) (Tabela 1).

Tabela 1 - produtos domissanitários frequentemente utilizados nos lares da população do bairro Parque São Camilo em Itu SP.

\begin{tabular}{lc}
\hline Saneantes domissanitários & Frequência de cada produto $(\mathbf{\%})$ \\
\hline Desinfetante & $\mathbf{1 0 0}$ \\
\hline Amaciante para tecidos & $\mathbf{9 5}$ \\
\hline Alvejante & $\mathbf{8 3}$ \\
\hline Mata-mosquitos em aerossol & $\mathbf{6 8}$ \\
\hline Sapólio & $\mathbf{3 0}$ \\
\hline Produto contra ratos & $\mathbf{1 5}$ \\
\hline Soda cáustica & $\mathbf{1 4}$ \\
\hline
\end{tabular}

Fonte: Autores.

Conforme esperávamos, os saneantes são frequentemente armazenados em locais inadequados, e entre os locais estão: embaixo do tanque de lavar roupas, no armário da cozinha ou banheiro (27\%), na parte de cima do armário da cozinha, fora do alcance das crianças (26\%), no quartinho dos fundos, numa prateleira baixa (10\%), próximo ao tanque, numa caixa, no chão da área de serviço (8\%), entre outros (29\%) (Tabela 2).

Tabela 2 - locais de armazenamento de domissanitários.

\section{Locais de armazenamento de domissanitários}

Na parte de cima do armário da cozinha, fora do alcance das crianças.

Embaixo do tanque de lavar roupas, no armário da cozinha ou banheiro.

No quartinho dos fundos, numa prateleira baixa.

Próximo ao tanque, numa caixa, no chão da área de serviço.

Outros
Frequência de armazenamento (\%)
27

10

29

Fonte: Autores.

Verificamos que, independentemente da idade, sexo, histórico de intoxicações e uso de EPI, o comportamento de ler o rótulo das embalagens mostrou-se associado à lavagem das mãos e ao não uso de produtos saneantes clandestinos $(\mathrm{p}=0,025)$ (Tabela 3). 
Tabela 3 - estratificação por sexo, faixa etária, histórico de intoxicação, uso de proteção individual, lavagem de mãos e uso de produto clandestino.

\begin{tabular}{|c|c|c|c|c|}
\hline \multirow[b]{2}{*}{ Variável dependente } & \multirow[b]{2}{*}{$\begin{array}{c}\mathrm{N}^{\circ} \\
\text { indivíduos }\end{array}$} & \multicolumn{2}{|c|}{ Variável independente } & \multirow{2}{*}{$\begin{array}{c}\begin{array}{c}\text { Exato de } \\
\text { Fisher }\end{array} \\
\text { Valor p }\end{array}$} \\
\hline & & Não lê rotulo & Lê rótulo & \\
\hline Feminino & 61 & 8 & 53 & 0,521 \\
\hline Masculino & 39 & 3 & 36 & \\
\hline 30-39 anos & 40 & 4 & 36 & 0,784 \\
\hline 40-49 anos & 29 & 3 & 26 & \\
\hline 50-59 anos & 21 & 2 & 19 & \\
\hline 60 anos ou mais & 10 & 2 & 8 & \\
\hline Sem histórico de intoxicação prévia & 82 & 8 & 74 & 0,412 \\
\hline Com histórico de intoxicação prévia & 18 & 3 & 15 & \\
\hline Não usa proteção/EPIs & 71 & 9 & 62 & 0,502 \\
\hline Usa proteção/EPIs & 29 & 2 & 27 & \\
\hline Lava as mãos e não utiliza produto clandestino & 60 & 3 & 57 & 0,025 \\
\hline Não lava as mãos, e utiliza produto clandestino & 40 & 8 & 32 & \\
\hline
\end{tabular}

Fonte: Autores.

\section{Discussão}

A partir das informações que foram obtidas durante a pesquisa foi possível constatar que a população do bairro Parque São Camilo em Itu - SP está bem informada e possui conhecimento sobre os riscos que estão associados ao uso de saneantes domissanitários. Assim, enquanto no estudo realizado por Souza e colaboradores (2015), 37\% dos 61 entrevistados já haviam vivenciado ou tomado conhecimento de casos de intoxicação por domissanitários; e, enquanto que na cidade de Juramento MG, Freitas e colaboradores (2012) evidenciaram que entre os 121 indivíduos que foram aleatoriamente entrevistados a intoxicação por saneantes domissanitários $(11,6 \%)$ foi mais frequente que os casos de intoxicação por medicamentos $(9,1 \%)$ e agrotóxicos (2,5\%); em nosso estudo, somente $18 \%$ dos 100 moradores que foram entrevistados informaram já ter presenciado ou se intoxicado com um produto domissanitário.

Além disso, diferentemente de Souza e colaboradores (2015), que no Complexo do Morro do Alemão demonstraram que $95 \%$ dos moradores responderam não lavar as mãos depois do contato com os saneantes - o que pode aumentar o número de intoxicações pela exposição a estes produtos - nossos resultados mostraram que o comportamento de leitura dos rótulos é bem frequente nos moradores do bairro Parque São Camilo em Itu - SP, e que a leitura está diretamente associada à lavagem das mãos e ao não uso de produtos saneantes clandestinos, independentemente de idade e sexo.

Vale ressaltar também que mesmo entre os entrevistados que não lavam as mãos após o uso de saneantes e entre os indivíduos que já presenciaram ou se intoxicaram com domissanitários foi evidenciado a prática de leitura de rótulos, o que pode implicar ou que os rótulos não trazem informações suficientes, conforme demonstrou Borges e colaboradores em 2019, ou que as pessoas não compreendem as informações de uso, segurança e perigo que estão contidas nos rótulos das embalagens (Pinheiro et al., 2014).

No Complexo do Morro do Alemão, os pesquisadores justamente constataram uma lacuna entre a leitura dos rótulos e o entendimento das informações que nele são veiculadas - isto porque muitos dos entrevistados, ainda que tenham informado realizar a leitura dos rótulos, não utilizam EPI adequados ou lavam as mãos após o uso de saneantes - e que as redações técnicas nos rótulos cumprem determinações técnicas legais, porém sem a preocupação de adaptação linguística para o entendimento do consumidor (Souza et al., 2015). 
Vale ressaltar aqui a importância da leitura dos rótulos, uma vez que alguns produtos são concentrados e precisam ser diluídos e/ou não podem ser misturados. Estas informações que estão contidas nos rótulos das embalagens certamente minimizam os casos de intoxicação por saneantes domissanitários aplicados em ambientes fechados ou não. $\mathrm{O}$ fato de que muitas pessoas realizam a prática de misturar alguns produtos saneantes, que por sua vez não poderiam ser misturados ou aplicados ao mesmo tempo, contribui para a ocorrência de intoxicações do manipulador e das pessoas e animais que estão próximos do ambiente exposto (Presgrave, 2007).

Um dos agravantes para intoxicação por domissanitários é a comercialização de produtos sem rótulo, que são comumente vendidos em garrafas do tipo PET, e que podem invariavelmente oferecer mais riscos à saúde do indivíduo exposto. 64\% da população do bairro Parque São Camilo relatou não utilizar produtos clandestinos e, ainda, lavar as mãos após o uso de domissanitários (60\%). A ANVISA por meio da Resolução da Diretoria Colegiada (RDC) nº 59/2010, proíbe o reaproveitamento de embalagens usadas de alimentos e bebidas para armazenamento de saneantes, e o material da embalagem que está em contato direto com o produto precisa necessariamente possuir uma composição adequada para que não ocorram reações químicas entre os produtos e as embalagens (ANVISA, 2010).

Contudo, ainda que a população avaliada tenha bons hábitos relacionados ao uso de domissanitários, há certas deficiências quanto ao armazenamento destes produtos. Nossos resultados demonstraram que, erroneamente, grande parte dos entrevistados armazenam os saneantes em locais inseguros ou impróprios e de fácil acesso às crianças, que representam o público mais suscetível a intoxicação, visto que têm ao seu alcance uma variedade de produtos chamativos com cores e formatos que se assemelham a brinquedos, e que são potencialmente nocivos à saúde, especialmente quando há exposição com a pele ou ingestão do produto. Assim, de acordo com a RDC 13/07 publicada pela ANVISA, as embalagens que sejam semelhantes a brinquedos, devem conter um lacre de segurança ou um componente que impeça a ingestão do mesmo (ANVISA 2007; 2010). Por fim, ainda que muitos armazenem de maneira inadequada, $26 \%$ dos entrevistados se atentaram a orientação contida nos rótulos: "conserve fora do alcance das crianças e dos animais domésticos", informação esta que, segundo a ANVISA, é obrigatória (ANVISA, 2012).

\section{Conclusão}

A pesquisa permitiu identificar que a população estudada no bairro Parque São Camilo adota comportamentos que estão alinhados ao uso seguro de saneantes, ainda que seu armazenamento seja inadequado. A leitura de rótulos pela população se associa com lavagem de mãos e não uso de produtos clandestinos, o que sugere que possivelmente o letramento geral da população e políticas públicas têm sido bem-sucedidas em promover a leitura de rótulos entre a população avaliada neste estudo. Ainda assim, o número de indivíduos que lê rótulos e não possui hábitos seguros de uso de saneantes é considerável, evidenciando ainda existir uma lacuna entre a informação contida nos rótulos e sua compreensão, o que demonstra que ainda há espaço para que políticas públicas visando à educação da população quanto ao uso de domissanitários sejam aprimoradas, consequentemente fortalecendo estratégias de segurança da população, do meio ambiente e de práticas de educação em saúde nas escolas e comunidades. Por fim, estudos como este são importantes para o desenvolvimento de uma panorama mais amplo e identificação de diferentes comportamentos de risco, formas de uso e armazenamento de saneantes domissanitários pela população brasileira.

\section{Agradecimentos}

Os autores agradecem a participação da população do bairro Parque São Camilo de Itu - SP e aos estudantes e professores do grupo de pesquisa Avaliação e Risco em Toxicologia (ARTox) da Universidade de Sorocaba, em especial a estudante Izadora Renosto. 


\section{Referências}

Agência Nacional de Vigilância Sanitária (2005). Resolução da Diretoria Colegiada $n^{\circ}$ 338. Para efeito deste regulamento, define-se como embalagem com gatilho aquela confeccionada em material plástico resistente e compatível com o produto, possuidora de gatilho propulsor, bico de jato contínuo ou spray e tubo pescante. Brasil: ANVISA. http://antigo.anvisa.gov.br/legislacao\#/visualizar/27668

Agência Nacional de Vigilância Sanitária (2007). Resolução da Diretoria Colegiada ${ }^{\circ}$ 13. Aprova o Regulamento Técnico para os produtos de limpeza e afins harmonizando no âmbito do Mercosul. Brasil: ANVISA. http://bvsms.saude.gov.br/bvs/saudelegis/anvisa/2007/rdc0013_28_02_2007.html.

Agência Nacional de Vigilância Sanitária (2010). Resolução da Diretoria Colegiada $n^{\circ}$ 59. Dispõe sobre os procedimentos e requisitos técnicos para a notificação e o registro de produtos saneantes e dá outras providências. Brasil: ANVISA. http://antigo.anvisa.gov.br/legislacao\#/visualizar/28612.

Agência Nacional de Vigilância Sanitária (2012). Orientações para os consumidores de saneantes. Brasília - DF: ANVISA. https://www.gov.br/anvisa/ptbr/assuntos/fiscalizacao-e-monitoramento/vigilancia-de-saneantes/arquivos/8600json-file-1/view

Agência Nacional de Vigilância Sanitária (2013). Resolução da Diretoria Colegiada $\mathrm{n}^{\circ}$ 47. Aprova o regulamento técnico de boas práticas de fabricação para produtos saneantes, e dá outras providências. Brasil: ANVISA. http://bvsms.saude.gov.br/bvs/saudelegis/anvisa/2013/rdc0047_25_10_2013.pdf

Agência Nacional de Vigilância Sanitária (2020). Instrução Normativa - IN n 70 . Dispõe sobre a inclusão de declaração sobre nova fórmula na rotulagem de saneantes quando da alteração de sua composição. Brasil: ANVISA. http://antigo.anvisa.gov.br/legislacao\#/visualizar/432068

Agência Nacional de Vigilância Sanitária (2021). Biblioteca de saneantes: atualizada em 21/01/2021. Brasil: ANVISA. https://www.gov.br/anvisa/ptbr/assuntos/regulamentacao/legislacao/bibliotecas-tematicas/arquivos/biblioteca-de-saneantes_portal.pdf

Almeida A. K. A., Oliveira E. S., Araújo I. G., Monteiro F. F. C., Albuquerque P. L. M. M., \& Romeu G. A. (2021). Intoxicações por domissanitários notificados no período de 2015 a 2019 no Estado do Ceará. Revista de Casos e Consultoria, 12(1), 1-15.

Barioni E. D., \& Profeta G. (2021). O livro das intoxicações: educação para a prevenção de acidentes. Eduniso.

Borges R. V., \& Diniz R. L. (2019). Avaliação das informações nas rotulagens de produtos saneantes domissanitários. Ergodesign E HCI, 7(1), 14-26.

Brasil. Ministério da Saúde (2020). Intoxicação exógena - notificações registradas no SINAN NET. http://tabnet.datasus.gov.br/cgi /tabcgi.exe?sinannet/cnv/Intoxsp.def.

Costa G. F. O., Filho S. D., Costa G. V., Faria A. A. S., Rodrigues H. C., \& Laval C. A. B. P. (2020). Intoxicações exógenas em menores de 15 anos notificadas no centro de informações toxicológicas de Goiás. Brasilian Journal of Health, 3(6), p.20070-20084.

Fundação Oswaldo Cruz (2020). Sistema Nacional de Informações Tóxico-Farmacológicas. FIOCRUZ. https://sinitox.icict.fiocruz.br/

Freitas R. F, Royo V. A, \& Moura P. M. S. (2012). Avaliação do risco de intoxicações por medicamentos, domissanitários e agrotóxicos na população de Juramento-MG. Conexão ciência, 7(1), 46-53.

Pinheiro G. A., Macedo I., Silva J. A., \& Janini M. J. D. M. (2014). Conscientização sobre o uso correto de saneantes domissanitários visando a prevenção de acidentes, intoxicações e contaminação ambiental. Revista Diálogos: Extensão e Aprendizagem: tempos e espaços, 19(1), 8-16.

Presgrave R. F. (2007). Avaliação das intoxicações acidentais humanas causadas por produtos saneantes domissanitários como subsídio para ações de vigilância sanitária [tese de doutorado]. Rio de Janeiro: Instituto Nacional de Controle de Qualidade em Saúde, Fundação Oswaldo Cruz.

Presgrave R. F., Camacho L. A. B., \& Villas Boas M. H. S. (2009). Análise dos dados dos centros de controle de intoxicações do Rio de Janeiro, Brasil, como subsídio às ações de saúde pública. Cad. Saúde Pública, 25(2), 401-409.

Rodrigues C. D. R. R. (2010). Comunicação de risco e comunicação publicitária de produtos saneantes domissanitários. Biblioteca on-line de ciências da comunicação. http://www.bocc.ubi.pt/pag/bocc-rodrigues-carla.pdf

Rodrigues F. P. M., Campos A. S. S., Moraes K. G. C., Costa M. M. R., Maia S. C., Pontes S. R. S., Silva W. N., \& Moraes F. C. (2021). Intoxicação Exógena: análise epidemiológica dos casos notificados em menores de cinco anos em São Luís - MA. Brasilian Journal of Development, 7(1), p. 9978-9995.

Souza R.O. L., Seixas Filho J. T., Miranda M. G., \& Carvalho Neto F. M. (2015). O Impacto dos produtos domissanitários na saúde da população do Complexo do Alemão-Rio de Janeiro. Revista Química nova na escola, 37(2), 93-97. 